\title{
Long-term Results of a Primary End-to-end Anastomosis in Peroperative Detected Bile Duct Injury
}

\author{
P. R. de Reuver • O. R. C. Busch • E. A. Rauws • \\ J. S. Lameris • Th. M. van Gulik • D. J. Gouma
}

Published online: 24 February 2007

(C) 2007 The Society for Surgery of the Alimentary Tract

\begin{abstract}
The management of a bile duct injury detected during laparoscopic cholecystectomy is still under discussion. An end-to-end anastomosis (with or without T-tube drainage) in peroperative detected bile duct injury has been reported to be associated with stricture formation of the anastomosis area and recurrent jaundice. Between 1991 and 2005, 56 of a total of 500 bile duct injury patients were referred for treating complications after a primary end-to-end anastomosis. After referral, $43(77 \%)$ patients were initially treated endoscopically or by percutaneous transhepatic stent placement $(n=3 ; 5 \%)$. After a mean follow-up of $7 \pm 3.3$ years, 37 patients $(66 \%)$ were successfully treated with dilatation and endoscopically placed stents. One patient died due to a treatment-related complication. A total of 18 patients (32\%) underwent a hepaticojejunostomy. Postoperative complications occurred in three patients $(5 \%)$ without hospital mortality. These data confirm that end-to-end anastomosis might be considered as a primary treatment for peroperative detected transection of the bile duct without extensive tissue loss. Complications (stricture or leakage) can be adequately managed by endoscopic or percutaneous drainage the majority of patients $(66 \%)$ and reconstructive surgery after complicated end-to-end anastomosis is a procedure with relative low morbidity and no mortality.
\end{abstract}

Keywords Cholecystectomy · Bile duct injury . End-to-end anastomosis

\section{Introduction}

Bile duct injury (BDI) after laparoscopic cholecystectomy (LC) is still a major problem in current surgical practice. BDI is associated with reduced survival, increased morbidity, and

This paper has been presented at the 47th SSAT Annual Meeting, May 20-24, 2006, in Los Angeles, California.

P. R. de Reuver • O. R. C. Busch • T. M. van Gulik •

D. J. Gouma $(\bowtie)$

Department of Surgery, Amsterdam Medical Center, Meibergdreef 9, 1105 AZ, Amsterdam, The Netherlands

e-mail: d.j.gouma@amc.uva.nl

\section{E. A. Rauws}

Department of Gastroenterology, Academic Medical Center, Amsterdam, Netherlands

J. S. Lameris

Department of Radiology, Academic Medical Center,

Amsterdam, Netherlands poor long-term quality of life $(\mathrm{QoL}))^{1,2}$ The incidence of BDI at laparoscopic cholecystectomy has been reported between 0.3 to $1.4 \%,{ }^{3-5}$ depending on the criteria used to define the injury as well as the study population. Of these injuries, one-third is detected during the procedure. ${ }^{6}$ Measures to prevent and recognize BDI are outlined in many publications..$^{6-8,9-11}$ The optimal treatment strategy and short- and long-term outcome has been published extensively. ${ }^{12-14}$ Controversy exists however about the management of peroperative detected BDI. The most important factor is the extent of tissue loss of the common bile duct, but also severity of inflammation and the size and diameter of the proximal duct. The peroperative management range from simple drainage and referral to a tertiary center to an end-toend anastomosis (EEA) (duct to duct, with or without T-tube drainage) or a hepaticojejunostomy (HJ).

It has been suggested that EEA is associated with a relative high stricture rate up to $70-80 \%$ and consequently a high incidence of secondary repair. ${ }^{15}$ Therefore, many tertiary centers prefer to perform a HJ instantly. A secondary repair after EEA should be associated with an increased risk of postoperative complications as the 
formation for strictures and stenosis. ${ }^{16,17}$ Others, however, consider EEA as a relative simple definitive repair, and also an optimal initial drainage procedure before reconstructive surgery in a secondary setting. ${ }^{18}$ Reports on large consecutive series to analyze the outcome of EEA are scary because this procedure is generally not performed in referral centers. One should realize that patients referred to such a center after previous EEA elsewhere are a negative selection of the EEA population. So far, a systematic analysis of a large group of patients with an EEA has not been performed and therefore this study was conducted.

The aim of the present study was to analyze short- and long-term outcome in patients who are referred after failure of a primary EEA.

\section{Patients and Methods}

\section{Patients Cohort and Data Collection}

Between January 1991 and January 2006, 500 consecutive patients were referred to the Academic Medical Center (AMC) in Amsterdam for the management of a BDI after cholecystectomy. Patient data was induced in a prospective database. All types of BDI were included, also minor injuries such as leakage from the cystic duct or ducts of Luschka. To define the location of BDI, the Bismuth classification was used. ${ }^{18}$ For the present study, the medical charts of all patients who underwent a primary EEA were retrospectively reviewed to analyze the initial operation reports and clinical data.

Data from the referring hospital included: indication for cholecystectomy, type of initial procedure, location of injury, type of repair including the use of a T-tube, the postoperative diagnostic interventions, and the therapeutic interventions before referral. Data from the present center included: symptoms at referral, diagnostic work-up, type of treatment, short-term, and long-term complications.

Endoscopic, Radiological, and Surgical Treatment for complicated EEA

Endoscopic treatment was performed by balloon dilatation or catheter dilatation before stent placement. The biliary stent is placed over the guide wire bridging the stenosis. Two or more stents were inserted if possible. For multiple stent insertion, an endoscopic sphincterotomy was performed to facilitate stent placement. Stents were replaced after 6 weeks and subsequently exchanged every 3 months to avoid cholangitis.

Percutaneous transhepatic catheterization was performed by injecting the contrast medium from the right intercostal approach. A right or left approach for the percutaneous transhepatic biliary drainage was chosen depending on ultrasound images illustrating the biliary anatomy, and the possibility of puncturing a dilated intrahepatic bile duct. Catheterization of intrahepatic bile ducts was performed in standard fashion. A guide wire was advanced through the biliary stricture into the duodenum. When this was achieved, a biliary drainage catheter was inserted. All drainage procedures were performed with the administration of broad-spectrum antibiotics.

In case of a surgical reconstruction, the procedure was performed via a Roux-en-Y hepaticojejunostomy. The stricture in the CBD is transected and the hilar plate is opened. The hepatic ducts of different segmental bile ducts are mobilized and from there opened over the left hepatic duct. Intrahepatic segmental ducts are mobilized and if possible sutured together before one or two jejunal anastomosis are made. A closed suction drain is placed during operation and removed 24-48 hours after surgery. Percutaneous transhepatic drains, when inserted before surgery are left in place and removed after 10 days till 6 weeks, depending on the clinical course, the level of anastomosis and the surgeons' preference.

\section{Outcome}

Follow-up data was obtained through outpatient records and the records of the general practitioner. The outcome of treatment was analyzed by the number complications and late restenosis during follow-up. Failure of treatment was defined as recurrent stenosis after stent therapy followed by surgery or recurrent stenosis after surgical reconstruction followed by additional therapy.

\section{Statistical Analysis}

Data from patient characteristics, management, and outcome show descriptive statistics in number of patients and percentages. Mean and median values are given with a minimum and maximum. Long-term stricture-free survival was analyzed by Kaplan Meier Survival Analysis. Data analyses were performed using SPSS ${ }^{\circledR}$ software (SPSS, Chicago, Illinois, USA).

\section{Results}

Patients' Characteristics at Referral

The referral pattern of BDI patients $(n=500)$ throughout the last 15 years are summarized in Fig. 1. From the total of 500 patients, $56(11.5 \%)$ underwent a primary EEA. Patient characteristics are listed in Table 1. The laparoscopic 
Figure 1 Referred patients for treatment of bile duct injury. Total number of referred patients (red), patients referred after a primary end to end anastomosis (blue), and patients referred after a primary biliodigestive reconstruction (green).

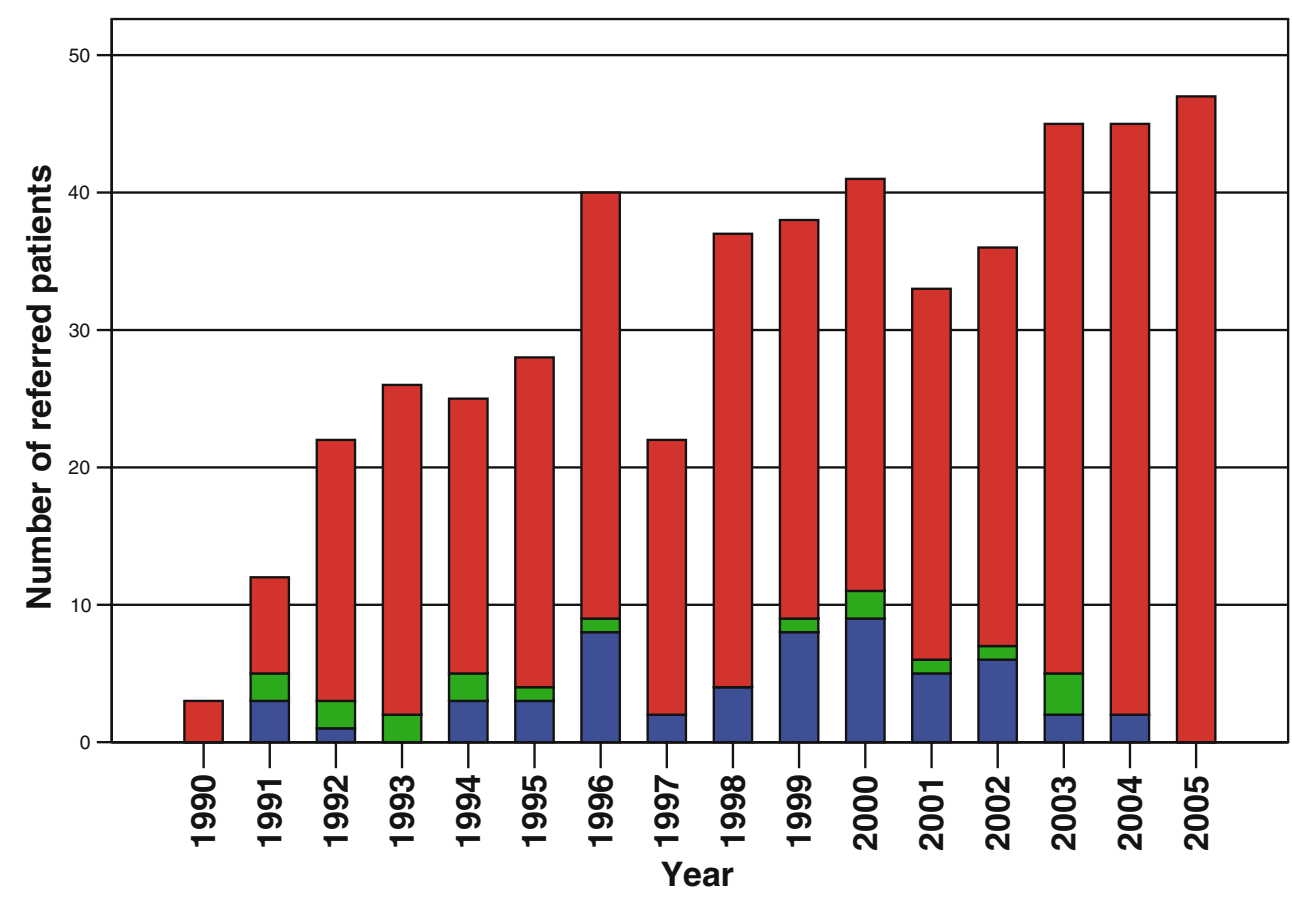

cholecystectomy $(n=48,86 \%)$ was converted in all patients. An open cholecystectomy was performed in eight patients $(14 \%)$. In 49 patients $(88 \%)$, the anastomosis was made over a T-tube. The tube was removed at the referring hospital or at the AMC after a mean of 52 days (range 2-145 days).

After the primary EEA, 19 patients (34\%) underwent other therapeutical interventions before referral (Table 2). These patients underwent a range of one to three procedures before referral (median 2). The interventions included a relaparotomy in two patients $(4 \%)$, percutaneous drainage of fluid collections in five patients $(9 \%)$, endoscopicially

Table 1 Patient Characteristics

\begin{tabular}{lll}
\hline & Primary EEA & \\
\cline { 2 - 3 } & $n=56$ & \\
\hline Age at cholecystectomy & & \\
$\quad$ Mean (years) & 52 & 77 \\
Gender & & \\
$\quad$ Female & 43 & 80 \\
Indication for cholecystectomy & 45 & 9 \\
$\quad$ Symptomatic cholelithiasis & 5 & 1 \\
Cholecystitis & 6 & 14 \\
Cholecystitis a froid & & 86 \\
Type of initial operation & 8 & 88 \\
$\quad \begin{array}{l}\text { Open procedure } \\
\text { Laparoscopic to open } \\
\text { procedure }\end{array}$ & 48 & \\
Anastomosis over T-tube & & \\
Duration of T-tube in situ & 49 & \\
Days, median (range) & $42(2-145)$ & \\
\hline
\end{tabular}

placed stents in 12 patients (21\%), a papillotomy in nine patients $(16 \%)$, and percutaneous transhepatic drainage in two patients $(4 \%)$. The median interval from the primary EEA to referral was 16 weeks (range $0-141$ weeks). At referral, a biliary stricture was diagnosed in 38 patients (68\%); in 10 patients (18\%), bile leakage was diagnosed and combination of both in eight patients (14\%). Symptoms at referral were cholestasis $(n=14,25 \%)$, cholangitis $(n=10$, $18 \%)$, and abdominal pain $(n=15,27 \%)$. Three patients were referred because of uncontrolled sepsis $(n=2)$ and peritonitis $(n=1)$. According to the Bismuth classification, the majority of injuries (leakage of stricture) $(n=47,84 \%)$ was located below the bifurcation. In nine patients $(16 \%)$, the injury (mostly strictures) involved the bifurcation or the right or left hepatic duct (i.e., Bismuth classification grades IV and V).

\section{Management after Referral}

Diagnostic work-up was performed by CT-scan $(n=9$; $16 \%)$, endoscopic cholangiography $(n=38 ; 68 \%)$, and transhepatic cholangiography $(n=9 ; 16 \%)$. The definitive treatment of BDI patients after EEA is shown in the flow diagram (Fig. 3). After work-up, three patients (5.3\%) were treated with percutaneous transhepatic cholangiographic drainage (PTCD) and 40 patients $(71.4 \%)$ were treated endoscopically. Thirteen patients (23\%) underwent reconstructive surgery after work-up; eight patients because of a complete stenosis of the $\mathrm{CBD}$, in three patients reconstructive surgery was performed after failure of stent therapy at the referring hospital and in two patients because of a percutaneous fistula and persistent bile leakage. 
Table 2 Referral Pattern

\begin{tabular}{lll}
\hline & Primary EEA & \\
\cline { 2 - 3 } & $n=56$ & $\%$ \\
\hline Time interval between injury and referral & & \\
Weeks, median (range) & $16(0-141)$ & \\
Intervention after EEA and before referral & & \\
Explorative relaparotomy & 2 & 4 \\
Percutaneous drainage & 5 & 9 \\
Endoscopic stenting & 12 & 21 \\
Endoscopic papillotomy & 9 & 16 \\
PTD & 2 & 4 \\
Symptoms at referral & & \\
Cholestasis & 14 & 25 \\
Cholangitis/fever & 10 & 18 \\
Abdominal pain & 15 & 7 \\
Abces/biloma & 4 & 5 \\
Uncontrolled sepsis/peritonitis & 3 & \\
Diagnosis at referral & & 68 \\
Stenosis & 38 & 18 \\
Leakage & 10 & 14 \\
Combination of stenosis and leakage & 8 & 16 \\
Location of injury at referral ${ }^{\text {b }}$ & & 38 \\
I & 9 & 4 \\
II & 21 & \\
III & 17 & \\
IV & 7 & \\
V & & \\
& &
\end{tabular}

${ }^{\text {a }}$ Percutaneous transhepatic drainage

${ }^{\mathrm{b}}$ According to Bismuth classification

\section{Radiological and Endoscopic Treatment}

Three patients were successfully treated by PTCD. In two patients, a stenosis was treated by transhepatic dilatation and in one patient, bile leakage was treated by external transhepatic stent insertion.

Forty patients $(71.4 \%)$ were treated endoscopically (Fig. 2). In 37 patients (66\%), stent insertion was successful and in three patients $(5 \%)$, adequate drainage succeeded by papillotomy. The median number of stent replacements was five (range 1-15) with a median duration of treatment of 359 days (range 39-1,355). Complications occurred in nine patients $(24 \%)$. Stent dislodgment $(n=3)$, clogging $(n=2)$, and cholangitis $(n=5)$ were mild complications and were successfully treated by stent exchange or administration of antibiotics. One severe complication occurred in a 75-yearold patient. After 4 years of stent therapy, the stent migrated and perforated the duodenum. Finally, the patient died due to multiple organ failure and sepsis.

\section{Surgical Treatment}

After referral and during the follow-up period, a new hepatobiliary anastomosis was performed by hepaticojejunostomy in 13 patients (23.2\%). Mean duration of hospital stay was $9.1 \pm 3.1$ days. Postoperative complications occurred in one patient $(7.6 \%)$ who underwent a PTC procedure after leakage of the anastomosis. No hospital mortality occurred in patients who underwent a reconstructive procedure after a previous EEA.

\section{Long-term Follow-up}

After a mean follow-up of $7.1 \pm 3.3$ years, seven patients (13\%) have died. One endoscopically treated patient died due to a complication of treatment as described above. The other patients died due to malignancy $(n=4)$ and myocardial infarct $(n=2)$.

The long-term results in patients treated with endoscopic and radiological treatment are as follows: from a total of 43 patients treated with endoscopic or PTCD procedures, $86 \%$ $(n=37)$ was successful. In three patients $(7 \%)$, signs of restenosis occurred after stent removal after 2, 3.5, and 4 months. Continued stent therapy was successful in all three patients. Five patients underwent reconstructive
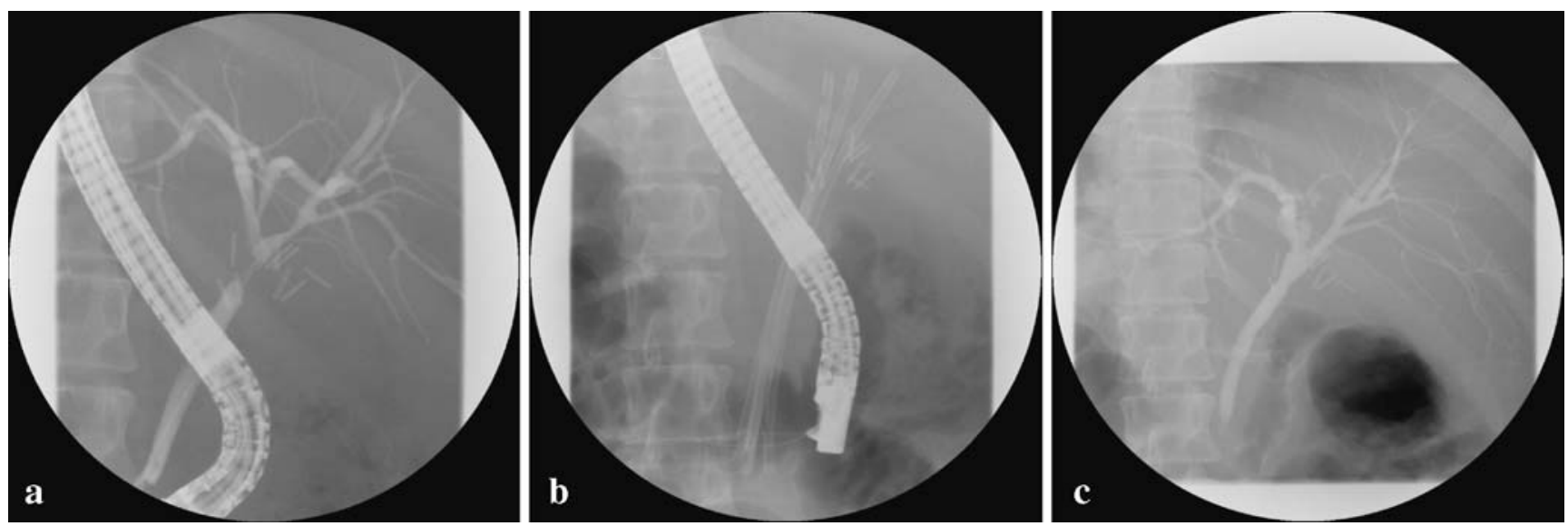

Figure 2 ERCP showing successful (aggressive) stent therapy after primary EEA. a Stenosis of the common bile duct. b Stents in situ. c After stent removal within a year. 
Figure 3 Flow diagram of the success and failure rates after a multidisciplinary treatment of patients who underwent a peroperative end to end anastomosis for bile duct injury. Given percentages are calculated from the number of patients in the previous flow box. PTCD Percutaneous transhepatic catheter dilatation.

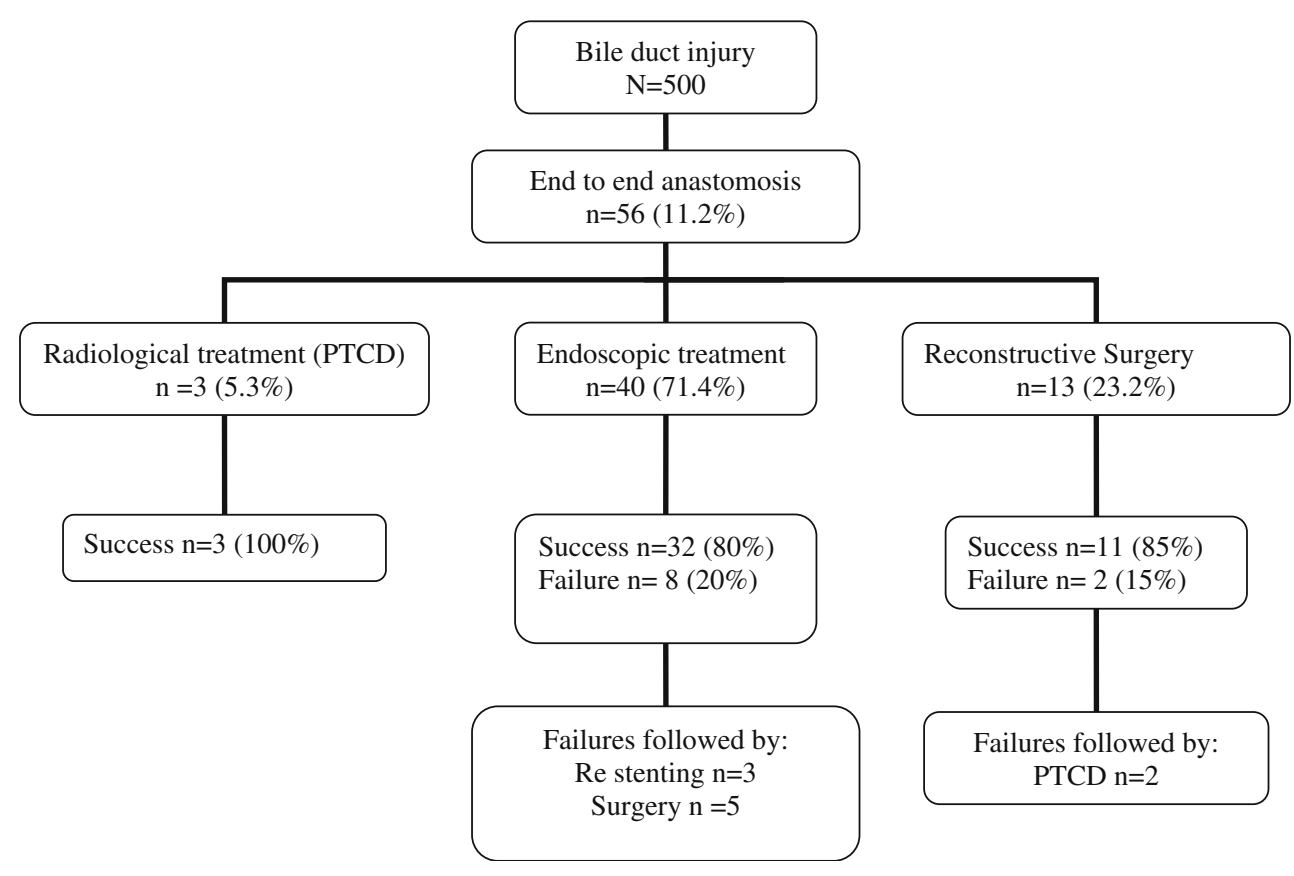

\section{Discussion}

surgery after prolonged endoscopic stenting. Postoperative complications occurred in two of the five patients and these patients received additional therapy for wound infection $(n=1)$ and postoperative cholangitis $(n=1)$.

The long-term results of surgical treatment after EEA are as follows; from 13 patients who underwent a HJ after work-up, a stenosis of the anastomosis occurred in two patients $(15 \%)$. Both patients underwent successful percutaneous transhepatic dilatation, respectively 9 and 35 months after surgery.

The overall 5 years stricture free survival in the total cohort $(n=56)$ is $91 \%$, shown by a Kaplan Meier curve in Fig. 4.

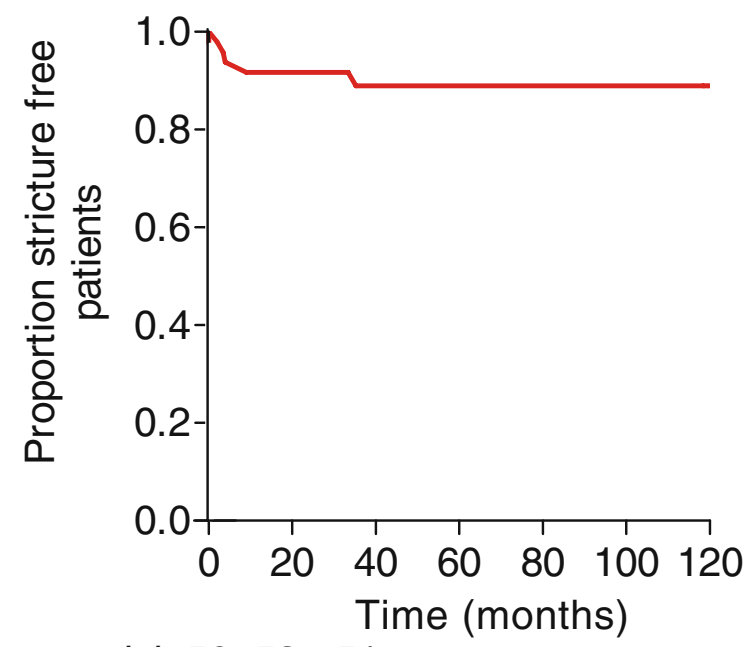

\section{patients at risk 565351}

Figure 4 Kaplan-Meier plot showing proportion of patients without restenosis among 56 bile duct injury patients treated for complications after EEA.
The present study describes a selected group of BDI patients, who were referred for treatment after a complicated EEA. This group of patients is a negative selection, representing the worst complications of EEA; otherwise, patients were not referred for additional treatment. So, this study does not provide any information about the success rate of EEA. The present study shows a long-term stricture free survival of $91 \%$ in EEA patients after treatment in a tertiary center. The analysis showed that even the majority of complications after primary AEE in a general hospital can successfully be treated by endoscopic and radiological interventions. In only one-third of the patients, a secondary surgical repair is necessary. The surgical reconstruction after EEA was associated with acceptable morbidity and without mortality.

Around 40 to 45 patients are referred annually without any sign of decrease over the last years. Considering 15.000 LC's per year in the Netherlands, we still consider 0.4$0.5 \%$ mentioned in the reviews as an underestimation of the real incidence of BDI, at least in the Netherlands. ${ }^{19}$ In $20 \%$ of the patients referred to the AMC, the injury was detected during the initial surgical procedure. This finding is similar to reports in literature. ${ }^{6,20}$ From the total of 500 referred BDI patients, $11.2 \%$ was referred for the treatment of a complication after peroperative EEA. Because referred patients only represent the complications after EEA, we do not know the real incidence of EEA procedures in BDI.

Peroperative repair in BDI detected during surgery can be performed by EEA (with or without the use of a T-tube) or by a primary HJ. A HJ is a more complex procedure and 
one should be very careful not to further extend the injury into the intrahepatic ducts or subsequently damage the arterial supply (bleeding and clipping or ligation of right hepatic artery). ${ }^{21,22}$ The present study shows that if complications occur after EEA, these can successfully be treated by percutaneous or endoscopic balloon dilatation and/or stenting in the majority of patients. A HJ in the acute setting without dilated bile ducts is even more difficult and therefore consulting a surgeon with experience in reconstructive hepatobiliary surgery is recommended. In contrast with a primary $\mathrm{HJ}$ an EEA is a relatively simple procedure and can also be performed in less experienced hands. The risk to increase damage is smaller in an EEA procedure and with the use of a $\mathrm{T}$ tube instant bile drainage is realized. If indicated, reconstructive surgery by means of an elective $\mathrm{HJ}$ can be performed. It is strongly advised to perform a HJ after classification the injury and analyzing the biliary anatomy. Preoperative cholangiography (with the use of the $T$ tube) will illustrate the location of the stenosis and the extension of dilation of the proximal bile ducts. A reconstructive procedure for stenosis of EEA has a satisfying outcome, as peroperative conditions are good after the inflammation has subsided and the bile ducts are dilated due to stenosis.

In a situation in which peroperative bile leakage is due to (extensive) tissue loss, in particular, in patients with more proximal lesions at the bifurcation or intrahepatically, no primary repair should be performed. In this situation, adequate drainage of the upper right abdomen is strongly advised and the patient should be referred for elective reconstruction. Referral to tertiary center in this situation has a positive effect on outcome. ${ }^{2}$

End-to-end anastomosis is reported to be associated with a high incidence of recurrent jaundice due to stricture formation of the anastomotic area. ${ }^{15}$ Therefore, some authors suggest that EEA is almost never appropriate if the bile duct has been completely transected, ${ }^{15,23}$ while others favor this strategy when there is no extensive tissue loss. ${ }^{18}$ Stent therapy for iatrogenic bile duct strictures has changed during the last decade and therefore the long-term outcome after stenting has improved. ${ }^{24}$ A more aggressive approach with more stents and smaller time intervals between stent changes is favored. With this new approach, $80 \%$ of the patients who undergo an ERCP for postoperative bile duct stenosis, have a 10-year stricture-free survival. ${ }^{25}$ Although complications occur at a significant rate, these are usually mild. The only severe complication occurred in the present series, due to a migrated stent, was not reported in previous series. ${ }^{24,25}$ After stent removal, recurrent stenosis develops in $20 \%$ of patients within 2 years of stent removal. ${ }^{25}$ Therefore, endoscopic treatment should be the initial management of choice for postoperative bile duct strictures. Without signs of improvement after endoscopic stenting, reconstructive surgery is indicated in otherwise fit patients.

Of interest is the evaluation of the long-term stricturefree survival after treatment for complications after EEA. After a mean follow-up of 7.1 years, restenosis after treatment developed in $9 \%$ of the patients. In all patients who underwent initial endoscopic therapy, restenosis occurred a relatively short time after stent removal, diagnosed within 2 to 8 months follow-up. Therefore, endoscopic treatment is not associated with a high rate of long-term restenosis after stent removal. In two patients, a restenosis occurred within 3 years after a hepaticojejunostomy. Symptoms were cholestasis and cholangitis. In both patients, transhepatic dilatation was successful to resolve the stenosis. The long-term stricture-free survival of $91 \%$ in the present series provides evidence for a good outcome after treating complicated EEA patients. If BDI is detected during surgery, in particular if there is no extensive tissue loss, the local anatomy is clear and there is no inflammation, EEA could be considered as a sufficient treatment strategy. Patients with postoperative complications (stricture or leakage) should be treated by a multidisciplinary team of gastroenterologists, radiologists, and surgeons. Postoperative complications can adequately be managed by endoscopic or percutaneous drainage in two-third of the patients. Reconstructive surgery after a complicated EEA is associated with low morbidity and no mortality.

Financial Support None.

Conflicts of Interest None.

\section{References}

1. Boerma D, Rauws EA, Keulemans YC, Bergman JJ, Obertop H, Huibregtse K, Gouma DJ. Impaired quality of life 5 years after bile duct injury during laparoscopic cholecystectomy: a prospective analysis. Ann Surg 2001;234:750-757.

2. Flum DR, Cheadle A, Prela C, Dellinger EP, Chan L. Bile duct injury during cholecystectomy and survival in Medicare beneficiaries. JAMA 2003;290:2168-2173.

3. Fletcher DR, Hobbs MS, Tan P, Valinsky LJ, Hockey RL, Pikora TJ, Knuiman MW, Sheiner HJ, Edis A. Complications of cholecystectomy: risks of the laparoscopic approach and protective effects of operative cholangiography: a population-based study. Ann Surg 1999;229:449-457.

4. Calvete J, Sabater L, Camps B, Verdu A, Gomez-Portilla A, Martin J, Torrico MA, Flor B, Cassinello N, Lledo S. Bile duct injury during laparoscopic cholecystectomy: myth or reality of the learning curve? Surg Endosc 2000;14:608-611.

5. MacFadyen BV, Jr., Vecchio R, Ricardo AE, Mathis CR. Bile duct injury after laparoscopic cholecystectomy. The United States experience. Surg Endosc 1998;12:315-321.

6. Lillemoe KD, Melton GB, Cameron JL, Pitt HA, Campbell KA, Talamini MA, Sauter PA, Coleman J, Yeo CJ. Postoperative bile 
duct strictures: management and outcome in the 1990s. Ann Surg 2000;232:430-441.

7. Strasberg SM. Avoidance of biliary injury during laparoscopic cholecystectomy. J Hepatobiliary Pancreat Surg 2002;9:543-547.

8. Chapman WC, Abecassis M, Jarnagin W, Mulvihill S, Strasberg SM. Bile duct injuries 12 years after the introduction of laparoscopic cholecystectomy. J Gastrointest Surg 2003;7:412-416.

9. Strasberg SM, Eagon CJ, Drebin JA. The "hidden cystic duct" syndrome and the infundibular technique of laparoscopic cholecystectomy - the danger of the false infundibulum. J Am Coll Surg 2000;191:661-667.

10. $\mathrm{Xu} \mathrm{F}, \mathrm{Xu} \mathrm{CG}, \mathrm{Xu} \mathrm{DZ}$. A new method of preventing bile duct injury in laparoscopic cholecystectomy. World J Gastroenterol 2004;10:2916-2918.

11. Way LW, Stewart L, Gantert W, Liu K, Lee CM, Whang K, Hunter JG. Causes and prevention of laparoscopic bile duct injuries: analysis of 252 cases from a human factors and cognitive psychology perspective. Ann Surg 2003;237:460-469.

12. Strasberg SM, Picus DD, Drebin JA. Results of a new strategy for reconstruction of biliary injuries having an isolated right-sided component. J Gastrointest Surg 2001;5:266-274.

13. Sicklick JK, Camp MS, Lillemoe KD, Melton GB, Yeo CJ, Campbell KA, Talamini MA, Pitt HA, Coleman J, Sauter PA, Cameron JL. Surgical management of bile duct injuries sustained during laparoscopic cholecystectomy: perioperative results in 200 patients. Ann Surg 2005;241:786-792.

14. Connor S, Garden OJ. Bile duct injury in the era of laparoscopic cholecystectomy. Br J Surg 2006;93:158-168.

15. Stewart L, Way LW. Bile duct injuries during laparoscopic cholecystectomy. Factors that influence the results of treatment. Arch Surg 1995;130:1123-1128.

16. Angel MM, Chan C, Orozco H, Barajas OA, Villalta JM,
Dominguez I, Erana J, Poucel F. Bile duct injuries related to misplacement of "T tubes". Ann Hepatol 2006;5:44-48.

17. Pellegrini CA, Thomas MJ, Way LW. Recurrent biliary stricture. Patterns of recurrence and outcome of surgical therapy. Am J Surg 1984;147:175-180.

18. Bismuth H, Majno PE. Biliary strictures: classification based on the principles of surgical treatment. World J Surg 2001;25:12411244.

19. Prismant. Utrecht, the Netherlands: National Medical Registration, 2005.

20. Carroll BJ, Birth M, Phillips EH. Common bile duct injuries during laparoscopic cholecystectomy that result in litigation. Surg Endosc 1998;12:310-313.

21. Buell JF, Cronin DC, Funaki B, Koffron A, Yoshida A, Lo A, Leef J, Millis JM. Devastating and fatal complications associated with combined vascular and bile duct injuries during cholecystectomy. Arch Surg 2002;137:703-708.

22. Nordin A, Makisalo $H$, Isoniemi $H$, Halme L, Lindgren L, Hockerstedt K. Iatrogenic lesion at cholecystectomy resulting in liver transplantation. Transplant Proc 2001;33:2499-2500.

23. Wudel LJ, Jr., Wright JK, Pinson CW, Herline A, Debelak J, Seidel S, Revis K, Chapman WC. Bile duct injury following laparoscopic cholecystectomy: a cause for continued concern. Am Surg 2001;67:557-563.

24. Costamagna G, Pandolfi M, Mutignani M, Spada C, Perri V. Long-term results of endoscopic management of postoperative bile duct strictures with increasing numbers of stents. Gastrointest Endosc 2001;54:162-168.

25. Bergman JJ, Burgemeister L, Bruno MJ, Rauws EA, Gouma DJ, Tytgat GN, Huibregtse K. Long-term follow-up after biliary stent placement for postoperative bile duct stenosis. Gastrointest Endosc 2001;54:154-161. 